EXTENDED REPORT

\title{
Neuro-ophthalmological disorders in HIV infected subjects with neurological manifestations
}

\author{
J-C Mwanza, L K Nyamabo, T Tylleskär, G T Plant
}

Br J Ophthalmol 2004;88:1455-1459. doi: 10.1136/bjo.2004.044289

See end of article for authors' affiliations .....................

Correspondence to: Jean-Claude Mwanza, University of Bergen, Centre for International Health, Armaver Hansen Building, N-5021 Bergen, Norway; jcmwanza@ hotmail.com

Accepted for publication 6 April 2004

\begin{abstract}
Aims: To determine the frequency and features of neuro-ophthalmological manifestations in neurologically symptomatic HIV infected patients and to assess whether or not the visual evoked potential (VEP) features in these patients differ from those of neurologically asymptomatic HIV infected patients.

Methods: Neuro-ophthalmological evaluation was performed in 166 neurologically symptomatic confirmed HIV positive patients, of whom 75 with normal ophthalmological examination were further studied by means of VEPs. The VEPs values were compared to those obtained from 53 other confirmed HIV positive subjects with neither ophthalmological nor neurological manifestations, who served as a comparison group and to the references values of our laboratory.

Results: An abnormal neuro-ophthalmological examination was noted in 99/166 patients (60\%). Eye movement disorders were present in 99 patients (51\%). Visual field defects were detected in $39 \%$ of the patients. Optic neuropathy was noted in $31 \%$, papilloedema in $27 \%$ and ocular motor nerve palsies in $26 \%$ of the patients. Toxoplasmosis and cryptococcosis were the most frequent associated pathologies, though in some patients the HIV itself was the presumed cause. VEPs were abnormal in $57 \%$ and $42 \%$ of patients with and without neurological manifestations, respectively. Compared to asymptomatic patients, symptomatic patients had a significantly increased mean latency; however, both groups had significant increase in mean latency compared to reference values.

Conclusion: Neuro-ophthalmological manifestations are common in neurologically symptomatic HIV infected patients. Subclinical dysfunction in the visual pathways is a common phenomenon in both HIV infected patients with and without neurological symptoms, but neurologically symptomatic patients seem to have more damage in their visual pathways.
\end{abstract}

$\mathrm{T}$ he human immunodeficiency virus (HIV) infection remains a major health problem in many developing countries. More than 40 million people are currently living with the HIV. ${ }^{1}$ Infection by HIV leads to a wide range of clinical pictures as it may involve almost all systems, including the nervous system and the eye. The nervous system is involved in up to $50 \%$ of the HIV infected subjects, resulting in different patterns of neurological involvement at some point during the infection. ${ }^{2-4}$ Additionally, histological studies have shown that $75-90 \%$ of AIDS patients incur damage to the brain, including the optic nerve. ${ }^{5}$ Furthermore, numerous reports have described HIV associated eye abnormalities, which affect $70-80 \%$ of all patients early or late during the course of their illness. ${ }^{6}$

Neuro-ophthalmological disturbances have been widely described in both asymptomatic HIV positive subjects and in those with full blown AIDS. In patients with AIDS there is a $3 \%-8 \%$ incidence of neuro-ophthalmological disorders. ${ }^{7-10}$ In addition, it has been shown that asymptomatic HIV infected subjects, even in early stages of the infection, exhibit ocular electrophysiological and psychophysical abnormalities. In recent years, there have been extensive studies on the ocular manifestations of AIDS, but only few of them have reported on systematic evaluation of the HIV/AIDS related neuroophthalmological manifestations, ${ }^{79}$ and only a few reviews have been published on this specific topic. ${ }^{11-13}$ Moreover, there are, to the best of our knowledge, no published reports specifically focusing on the prevalence and clinical features of the neuro-ophthalmological manifestations in neurologically symptomatic HIV infected patients. Thus, we wished (1) to determine the prevalence and features of neuro-ophthalmological manifestations in neurologically symptomatic HIV infected patients, and (2) to determine whether or not the visual evoked potential (VEP) features in HIV infected patients with neurological symptoms differ from those of HIV infected patients without neurological symptoms.

\section{SUBJECTS AND METHODS \\ Subjects}

The participants were all recruited from the Department of Neurology and the Unit of Infectious Diseases of the Kinshasa University Hospital in Kinshasa, from August to December 2001 and from July to December 2002. They consisted of 166 confirmed HIV infected patients (91 males and 75 females, mean age 39.5 (SD 8.7) years, age range 2061 years) with neurological manifestations (group I) and 53 other confirmed HIV infected subjects (28 males and 25 females, mean age 39.9 (6.2) years, age range 27-53 years) without any neurological manifestations (group II) or ophthalmological complaints at the time of the study. Neuro-ophthalmological examination was performed in all patients with neurological manifestations. In addition, VEPs were recorded in 75 patients of those with neurological manifestations (symptomatic patients) and in all 53 patients without neurological manifestations (asymptomatic patients) who served as a comparison group, with the understanding that they all had visual acuity of 0.8 or better, no ophthalmoscopic detectable abnormalities, and no visual field defects. Another group of healthy controls previously investigated served as the laboratory reference material. ${ }^{14}$

Abbreviations: ADC, AIDS related dementia complex; CRY, cryptococcosis; HIV, human immunodeficiency virus; LPH, lymphoma; TBC, tuberculosis; TXP, toxoplasmosis; VEP, visual evoked potential 


\section{Methods}

\section{Ophthalmological examination}

A routine neuro-ophthalmological examination was performed in all symptomatic patients. Visual acuity was measured with a Snellen chart and objective refraction was assessed using retinoscopy and Javal keratometry. The pupils were checked for size, reactivity to light, and near targets. Eyelids were examined to search for possible ptosis and retraction. Ocular motility (ductions and versions) was tested in each of the cardinal positions of gaze. Saccades were qualitatively assessed using a home made device consisting of two alternating light spots horizontally separated $50 \mathrm{~cm}$ apart in front of the subject. Pursuit eye movements were checked by asking the patient to maintain the fixation on a light spot moving slowly and horizontally from right to left and vice versa in front of him. Colour vision performance was assessed with Ishihara plates, and ocular fundus was examined by direct ophthalmoscopy through dilated pupils (tropicamide eye drops $1 \%$ ) with special attention drawn on the sharpness of the margin and the colour of the optic disc. A Goldmann perimeter was used to assess the visual fields.

\section{VEP recording procedure}

VEP recordings were obtained monocularly using the following settings: stimulus number $=128$, analysis period $=300 \mathrm{~ms}$, band pass $=1-70 \mathrm{~Hz}$ on a Cadwell 5200A, USA device. The visual stimulus was a pattern reversal checkerboard displayed on a 14 inch black and white monitor (Philips, Italy), placed at $1 \mathrm{~m}$ from the patient. Checks were oblong and each check measured 1.71 degrees of visual angle horizontally and 0.85 degrees vertically. The checkerboard had a $90 \%$ contrast with a luminance of $2 \mathrm{~cd} / \mathrm{m}^{2}$ and $90 \mathrm{~cd} /$ $\mathrm{m}^{2}$ for the black and white checks, respectively. Room lighting was kept constant during the examination $(5 \mathrm{~cd} /$ $\mathrm{m}^{2}$ ). Cortical responses were recorded using silver chloride electrodes placed over the occipital cortex $2 \mathrm{~cm}$ above the inion for the active, in the midline $2 \mathrm{~cm}$ anteriorly for the reference. The mid-frontal electrode was used as ground. Responses to 100 reversals were averaged. The P100 component of the cortical response and the peak to peak N75-P100 amplitude were considered for measurement. P100 latency values of the 75 patients in group I were compared to those in group II using the Student's $t$ test. The values obtained from these groups were further compared to the reference values of our laboratory.

\section{RESULTS}

The clinical examination included 166 HIV infected patients with various neurological manifestations. Their repartition according to the cause of neurological disturbances was as follows: cerebral toxoplasmosis 77 (46\%), cryptococcal meningitis $46(28 \%)$, tuberculous meningitis 23 (14\%), AIDS related dementia complex (ADC) $14(8 \%)$, cerebral lymphoma four (2\%), and herpes zoster two (1\%).

The neuro-ophthalmological manifestations are outlined in table 1. Overall, an abnormal examination was found in 99 patients $(60 \%)$. Among these patients, we found ocular movement abnormalities in 85 patients, visual field defects in 60 patients, optic neuropathy in 52 patients, papilloedema in 45 patients, and ocular nerve palsies including conjugate gaze and palsy convergence deficiency in 43 patients. Less common findings included upper eyelid retraction secondary to palsy of the seventh cranial nerve and cortical blindness.

Of the 85 patients with eye movement disturbances, abnormal saccades and abnormal eye pursuits were observed in 71 and 65 patients or $43 \%$ and $39 \%$ of the study population, respectively. Most of these 85 patients (53 patients or $62 \%$ ) had both abnormal saccades and abnormal pursuit eye movements while 18 and 12 patients had only abnormal saccades and abnormal pursuits, respectively. Saccades were slow in all patients and delayed in initiation in most of them. Only two patients presented with opposite way saccades. Abnormal pursuit eye movements consisted of saccadic movements.

Visual field defects were present in 39\% of the 153 subjects in whom the test was performed. Both visual field deficits consistent with retinal pathology, damage of the anterior and posterior visual pathways, were observed.

Fifty two patients had optic neuropathy that presented clinically as neuroretinitis, anterior or retrobulbar optic neuropathy. Most of these patients $(n=30)$ had unilateral involvement.

Ocular motor nerve palsies involving the abducens (17\%) and the oculomotor (9\%) were the most frequent. Unilateral involvement and isolated form were predominant.

Table 1 also shows the causes of the different neuroophthalmological manifestations in this series of patients. Toxoplasmosis was the predominant cause associated with optic neuropathy (40\%). Most cases of third cranial nerve palsy $(47 \%)$, sixth cranial nerve palsy (39\%), and visual field defect $(35 \%)$ were seen in patients with cryptococcosis while

Table 1 Frequency and aetiology of neuro-ophthalmological manifestations in $166 \mathrm{HIV}$ positive patients with neurological manifestations

\begin{tabular}{|c|c|c|c|c|c|c|c|}
\hline Manifestations & Total (\%) & $\operatorname{TXP}(n=77)$ & CRY $(n=47)$ & $\mathrm{TBC}(\mathrm{n}=23)$ & $\mathrm{LPH}(\mathrm{n}=4)$ & $H Z V(n=2)$ & HIV only $(n=14)$ \\
\hline Any manifestation & $99(60)$ & 35 & 32 & 15 & 3 & 2 & 12 \\
\hline Optic neuropathy & $52(31)$ & & & & & & \\
\hline Neuroretinitis & $25(15)$ & 17 & - & 2 & - & 1 & 5 \\
\hline Anterior optic neuropathy & $16(10)$ & 3 & 3 & 5 & - & - & 5 \\
\hline Retrobulbar optic neuropathy & $11(7)$ & 1 & 6 & 1 & - & 1 & 2 \\
\hline Eye movement disorders & $85(51)$ & & & & & & \\
\hline Abnormal saccades & 71 (43) & 27 & 18 & 12 & 2 & 2 & 10 \\
\hline Abnormal pursuits & $65(39)$ & 24 & 17 & 10 & 2 & 1 & 11 \\
\hline Nystagmus & $2(1)$ & 1 & - & - & 1 & - & - \\
\hline Ocular motor palsy & $43(26)$ & & & & & & \\
\hline IIIrd cranial nerve & $15(9)$ & 4 & 7 & 1 & 1 & 1 & 1 \\
\hline IVth cranial nerve & $2(1)$ & - & 1 & - & - & - & 1 \\
\hline Vlth cranial nerve & $28(17)$ & 7 & 13 & 4 & 2 & - & 2 \\
\hline Convergence insufficiency & $1(1)$ & - & 1 & - & - & - & - \\
\hline Supranuclear gaze palsy & $2(1)$ & 2 & - & - & - & - & - \\
\hline Visual field defects & 60 (39) & 19 & 21 & 10 & 3 & - & 7 \\
\hline Papilloedema & $45(27)$ & 10 & 11 & 10 & 3 & 1 & 10 \\
\hline Optic atrophy & $8(5)$ & 1 & 4 & - & 2 & 1 & - \\
\hline Cortical blindness & $1(1)$ & 1 & - & - & - & - & - \\
\hline
\end{tabular}


Table 2 VEP features in HIV infected patients with neurological manifestations (symptomatic patients), without neurological manifestations (asymptomatic patients), and laboratory reference values

\begin{tabular}{llll}
\hline & $\begin{array}{l}\text { Symptomatic patients } \\
(\mathbf{n}=75)\end{array}$ & $\begin{array}{l}\text { Asymptomatic patients } \\
(\mathbf{n}=53)\end{array}$ & References \\
\hline Abnormal VEPs, $n(\%)$ & $43(57 \%) \neq$ & $22(42 \%)$ & $100(5)$ \\
Mean latency (SD) (ms) & $115(14)^{*}, \dagger$ & $109(9)^{*}$ & $15(5)$ \\
Mean amplitudes (SD) ( $\mu \mathrm{V})$ & $8(3)^{*}, \dagger$ & $9(5)^{*}$ & \\
\hline *Significantly different from reference values, tsignificantly different from asymptomatic patients, fnot significantly \\
different from asymptomatic patients.
\end{tabular}

most cases of abnormal saccades (71\%) and pursuits (78\%) were observed in patients in whom the HIV itself was the presumed aetiology of neurological symptoms. Half of patients with optic atrophy had cryptococcosis. Cortical blindness was observed in a single patient with cerebral toxoplasmosis.

The results of the VEP recordings are summarised in table 2. Altogether, VEPs were abnormal in 57\% of HIV positive patients with neurological symptoms and in $42 \%$ of those without neurological symptoms. Although there was a trend that symptomatic patients are more likely to have abnormal VEPs, this trend was not statistically significant $\left(\chi^{2}=3.11, p=0.07\right)$. In the symptomatic patients VEPs were abnormal in $8 / 11$ patients $(73 \%)$ with HIV itself as the presumed cause of neurological manifestations, in $11 / 18$ patients $(61 \%)$ with cryptococcosis, in $7 / 13$ patients $(54 \%)$ with tuberculosis, and in $17 / 33$ patients (52\%) with toxoplasmosis. Logistic regression analysis revealed that none of the aetiologies significantly influenced the VEP. Only $2 / 75$ (3\%) of symptomatic patients and $1 / 43(2 \%)$ of the asymptomatic patients had unilateral alteration of VEP. Both groups showed a significant increase of the mean P100 latency compared to the reference values obtained in our laboratory. Symptomatic patients had a significantly prolonged mean P100 latency compared to the asymptomatic patients $(p=0.01)$. There was no significant difference in the mean amplitude of the two groups of HIV patients $(p=0.17)$, though both showed a significant decrease compared to the reference values $(p<0.05)$. In both groups of HIV patients no correlation (symptomatic patients: $r=-0.029$, asymptomatic patients: $r=-0.020$ ) could be found between the P100 latency and the amplitude.

\section{DISCUSSION}

This study has generated two types of results. Firstly, it was found that $60 \%$ of HIV patients with neurological symptoms have neuro-ophthalmological manifestations on clinical examination, which means that neurologically symptomatic HIV infected patients commonly exhibit neuro-ophthalmological manifestations. Secondly, VEPs were abnormal in 57\% of HIV positive patients with neurological manifestations and in $42 \%$ of those without neurological manifestations.

There are some limitations that need to be considered, especially regarding the distribution of aetiologies in the present series of patients. In contrast with the developed world where the incidence of neurological complications decreases following the introduction of highly active antiretroviral therapy, ${ }^{15}{ }^{16}$ the incidence of CNS related HIV pathologies are increasing in sub-Saharan Africa because these drugs are not available. It is therefore likely that the prevalence of HIV related neuro-ophthalmological manifestations found in this study is high in comparison to that reported in Western countries. Some other factors such as the lack of both prophylaxis against opportunistic infections and appropriate complementary tests may have a role in the observed prevalence. Since the magnitude of HIV associated neuro-ophthalmological complications in sub-Saharan Africa is largely not known, the results of the present study may be considered as a starting point for more studies in the future.

The relation between HIV infection and the occurrence of neuro-ophthalmological complications has been established for some years. Indeed, several studies have shown that patients with full blown AIDS or symptomatic HIV infection frequently exhibit neuro-ophthalmological manifestations, which result from central nervous system (CNS) opportunistic infections and neoplasms as well as the direct effect of the virus itself acting alone or in combination with other cofactors yet to be determined. ${ }^{79}{ }^{11}$ However, only very few large studies have specifically assessed the relative prevalence of the different neuro-ophthalmological disorders, which has been reported to range between $3 \%$ and $8 \% .{ }^{79}$ Surprisingly, there has not been a single report of such disorders in the subgroup of neurologically symptomatic AIDS patients. The difference in the homogeneity of the study population explains the huge difference in the prevalence of the neuroophthalmological manifestations. The high prevalence found in the present study indicates that HIV patients with neurological symptoms are at higher risk for neuro-ophthalmological complications.

Optic neuropathies have been described in HIV patients. They may be caused by a variety of pathologies including infectious, compressive and inflammatory processes. Of interest is the optic neuropathy related to the HIV itself. ${ }^{17}{ }^{18}$ In this series HIV was assumed to induce primary optic neuropathy in seven patients, representing $4 \%$ of the study population and $7 \%$ of patients with neuro-ophthalmological manifestations. The diagnosis was made by exclusion, in the absence of both any ophthalmoscopically observable retinopathy and any other cause of optic neuropathy. There is currently enough evidence that the optic nerves of HIV infected patients can undergo chronic degeneration resulting in axonal loss. ${ }^{10} 19$ Despite the enormous amount of research devoted to neurodegeneration in HIV infection, there remain a number of unclarified questions in relation to the mechanism by which HIV induces primary optic neuropathy. The current widely accepted theory emphasises the key role of tumour necrosis factor alpha (TNF- $\alpha$ ) in the genesis of primary HIV optic neuropathy. ${ }^{20}{ }^{21}$ In HIV dementia, which is a good example of axonal death in HIV patients and where the pathogenesis of neuronal damage has been widely investigated, the damage has been ascribed at least partly to the combined effect of neurotoxic agents including viral proteins and neurotoxic factors released from activated microglia and macrophages. ${ }^{52}$ Thus, our opinion is that primary optic neuropathy may result from the combination of both mechanisms, with the understanding that the mechanism having the key role may vary depending on factors yet to be determined by further studies.

In this study, eye movement disorders (abnormal saccades and pursuits) were the most frequent manifestations regardless of the aetiology. They were present in half of the study population and in $86 \%$ of patients with 
neuro-ophthalmological complications. This finding is consistent with the result of previous clinical and electrophysiological studies, which have shown that abnormal saccades and smooth pursuits are present in HIV infected subjects including those without any other clinical manifestation of HIV infection..$^{23-27}$ These ocular movement disturbances are believed to be more frequent in subjects with HIV dementia. Some investigators have postulated that in asymptomatic HIV subjects they represent early manifestations of HIV dementia. ${ }^{28}$ In our series $12 / 14$ patients with HIV dementia had abnormal saccades and pursuits. Besides, many others without dementia but with other HIV related CNS diseases exhibited the same manifestations. Whether or not all cases of ocular movements disorders are exclusively caused by HIV itself is therefore questionable, as concurrent opportunistic infections and other factors yet to be elucidated may have a role in the genesis of such abnormalities. Most patients with eye movement disturbances had both abnormal saccades and pursuits. This, together with the high prevalence of eye movement disturbances, suggests that the anatomical structures involved in the control of saccades and smooth pursuits are frequently involved in HIV infected subjects.

The prevalence of ocular nerve palsy found in the present study is $25 \%$. In the series reported by Helweg-Larsen et al, ${ }^{29}$ only 17 of 589 patients with neurological manifestations (3\%) had ocular nerve palsy. The prevalence of such disorders in patients with AIDS, regardless of the presence of neurological symptoms, has been reported to range between 3\% and $8 \% .^{930} 31$ Similarly to previous reports, ${ }^{7932}$ palsies involving the sixth and third cranial nerves were the most frequent in the present study, and toxoplasmosis and cryptococcosis were the two most common causes. The sixth cranial nerve has a long course, which makes it more vulnerable, especially to high intracranial pressure. The trochlear nerve, though the longest of the ocular motor nerves, seems to be rarely affected. It is however worth stressing that in a number of cases the exact cause of cranial nerve palsy is complicated by the presence of concurrent multiple potential underlying aetiologies.

VEPs were abnormal in $57 \%$ and $42 \%$ of patients with and without neurological symptoms, respectively. In HIV infected subjects subclinical abnormal VEPs have been reported to range from $3 \%$ to $49 \%,{ }^{33-36}$ though completely normal VEPs in both neurologically symptomatic and asymptomatic HIV infected subjects have also been found. ${ }^{37}$ Interestingly, the number of subjects with abnormal VEPs did not differ significantly in the two groups. These findings may indicate that HIV positive patients with neurological symptoms are not at higher risk for optic nerve damage than those without neurological manifestations. They further support both the hypothesis according to which axonal loss of the optic nerve is a common process occurring in all HIV infected subjects ${ }^{38} 39$ and the results of previous histological studies. ${ }^{10}{ }^{19}$ The significant difference observed between the mean latencies of subjects with and those without neurological symptoms may suggest that the earlier have more damage in the visual pathways compared to the latter. There is currently considerable evidence suggesting that, despite normal visual acuity, HIV infected subjects may have subclinical dysfunction of the optic nerves/retrochiasmal visual pathways that can be detected using electrophysiological methods. ${ }^{33}{ }^{35}$ Whether alteration of electrophysiology found in this study originates only from the optic nerve dysfunction remains unclear, since a loss of cortical neurons has been found in asymptomatic HIV positive subjects in early stages of the infection. ${ }^{40}$ These lesions as well as others affecting the retrogeniculate part of the visual pathways, especially the primary visual cortex, may result in delay in both cortical processing and signal generation and thus in VEP abnormalities.
In conclusion, neuro-ophthalmological manifestations are various and common in neurologically symptomatic HIV infected patients in sub-Saharan Africa. They are mainly caused by opportunistic infections, among which toxoplasmosis and cryptococcosis are the most common. The VEP results indicate that subclinical dysfunction in the visual pathways is a common phenomenon in both HIV infected patients with and without neurological symptoms, but neurologically symptomatic patients seem to have more damage to their visual pathways.

\section{ACKNOWLEDGEMENTS}

The Norwegian Educational Loan Fund and the University of Bergen, Norway, supported this study. We wish to thank Dr José Nkoy at the Unit for Infectious Diseases, Department of Internal Medicine, Kinshasa University Hospital, for assistance in tracing the patients.

\section{Authors' affiliations}

J-C Mwanza, Department of Ophthalmology, Kinshasa University Hospital, Kinshasa, Democratic Republic of Congo

L K Nyamabo, Department of Neurology, Kinshasa University Hospital, Kinshasa, Democratic Republic of Congo

J-C Mwanza, T Tylleskär, Centre for International Health, University of Bergen, Bergen, Norway

G T Plant, Department of Neuro-ophthalmology, The National Hospital for Neurology and Neurosurgery and Moorfields Eye Hospital, London, UK

\section{REFERENCES}

1 UNAIDS. Annual report, December 2003.

2 Gongora-Rivera F, Santos-Zambrano J, Moreno-Andrade T, et al. The clinical spectrum of neurological manifestations in AIDS patients in Mexico. Arch Med Res 2000;31:393-8.

3 Perriens JH, Mussa M, Luabeya MK, et al. Neurological complications of HIV1-seropositive internal medicine inpatients in Kinshasa, Zaire. J Acquir Immune Defic Syndr 1992;5:333-40.

4 Levy RM, Bredesen DE, Rosenblum ML. Neurologic complications of HIV infection. Am Fam Physician 1990;41:517-36.

5 Gray F, Adle-Biassette H, Chretien F, et al. Neuropathology and neurodegeneration in human immunodeficiency virus infection. Pathogenesis of HIV-induced lesions of the brain, correlations with HIV-associated disorders and modifications according to treatments. Clin Neuropathol 2001;20:146-55.

6 Jabs D, Quinn T. Acquired immunodeficiency syndrome. In: Pepose J, Holland G, Wilhelmus K, eds. Ocular infection and immunity. St Louis: MosbyYear Book, 1996:289-310.

7 Keane JR. Neuro-ophthalmologic signs of AIDS: 50 patients. Neurology 1991;41:841-5.

8 Jabs DA, Green WR, Fox R, et al. Ocular manifestations of acquired immune deficiency syndrome. Ophthalmology 1989;96:1092-9.

9 Mansour AM. Neuro-ophthalmic findings in acquired immunodeficiency syndrome. J Clin Neuroophthalmol 1990;10:167-74.

10 Sadun AA, Pepose JS, Madigan MC, et al. AIDS-related optic neuropathy: a histological, virological and ultrastructural study. Graefes Arch Clin Exp Ophthalmol 1995;233:387-98.

11 Currie J. AIDS and neuro-ophthalmology. Curr Opin Ophthalmol 1995;6:34-40.

12 Friedman DI. Neuro-ophthalmic manifestations of human immunodeficiency virus infection. Neurol Clin 1991;9:55-72.

13 Woods AD, Caputo MK. Neuro-ophthalmic manifestations of AIDS. Optom Clin 1996;5:113-52.

14 Mwanza JC, Lysebo D, Kayembe DL, et al. Visual evoked potentials in konzo, a spastic paraparesis of acute onset in Africa. Ophthalmologica 2003;217:381-6

15 Maschke M, Kastrup O, Esser S, et al. Incidence and prevalence of neurological disorders associated with HIV since the introduction of highly active antiretroviral therapy (HAART). J Neurol Neurosurg Psychiatry 2000;69:376-80

16 Sacktor N, Lyles RH, Skolasky R, et al. HIV-associated neurologic disease incidence changes: Multicenter AIDS Cohort Study, 1990-1998. Neurology 2001;56:257-60

17 Goldsmith P, Jones RE, Ozuzu GE, et al. Optic neuropathy as the presenting feature of HIV infection: recovery of vision with highly active antiretroviral therapy. Br J Ophthalmol 2000;84:551-3.

18 Larsen M, Toft PB, Bernhard $P$, et al. Bilateral optic neuritis in acute human immunodeficiency virus infection. Acta Ophthalmol Scand 1998;76:737-8.

19 Tenhula WN, Xu SZ, Madigan MC, et al. Morphometric comparisons of optic nerve axon loss in acquired immunodeficiency syndrome. Am J Ophthalmol 1992; 113:14-20.

20 Madigan MC, Sadun AA, Rao NS, et al. Tumor necrosis factor-alpha (TNFalpha)-induced optic neuropathy in rabbits. Neurol Res 1996;18:176-84. 
21 Lin XH, Kashima $Y$, Khan $M$, et al. An immunohistochemical study of TNFalpha in optic nerves from AIDS patients. Curr Eye Res 1997;16:1064-8.

22 Kolson DL, Lavi E, Gonzalez-Scarano F. The effects of human immunodeficiency virus in the central nervous system. Adv Virus Res 1998;50:1-47.

23 Johnston JL, Miller JD, Nath A. Ocular motor dysfunction in HIV-1-infected subjects: a quantitative oculographic analysis. Neurology 1996;46:451-7.

24 Friedman DI, Feldon SE. Eye movement abnormalities in the AIDS dementia syndrome. Neurology 1989;39:355.

25 Merrill PT, Paige GD, Abrams RA, et al. Ocular motor abnormalities in human immunodeficiency virus infection. Ann Neurol 1991:30:130-8.

26 Castello E, Baroni N, Pallestrini E. Neurotological auditory brain stem response findings in human immunodeficiency virus-positive patients withou neurologic manifestations. Ann Otol Rhinol Laryngol 1998;107:1054-60.

27 Sweeney JA, Brew BJ, Keilp JG et al. Pursuit eye movement dysfunction in HIV-1 seropositive individuals. J Psychiatry Neurosci 1991;16:247-52.

28 Currie J, Benson E, Ramsden B, et al. Eye movement abnormalities as a predictor of the acquired immunodeficiency syndrome dementia complex. Arch Neurol 1988;45:949-53.

29 Helweg-Larsen S, Jakobsen J, Boesen F, et al. Neurological complications and concomitants of AIDS. Acta Neurol Scand 1986;74:467-74.

30 Freeman WR, Lerner CW, Mines JA, et al. A prospective study of the ophthalmologic findings in the acquired immune deficiency syndrome. Am J Ophthalmol 1984;97:133-42.

31 Palestine AG, Rodrigues MM, Macher AM, et al. Ophthalmic involvement in acquired immunodeficiency syndrome. Ophthalmology 1984;91:1092-9.
32 Engstrom JW, Lewis E, McGuire D. Cranial neuropathy and and the acquired immunodeficiency syndrome. Neurology 1991;41:374.

33 Malessa R, Agelink MW, Diener HC. Dysfunction of visual pathways in HIV-1 infection. J Neurol Sci 1995;130:82-7.

34 Pierelli F, Soldati G, Zambardi P, et al. Electrophysiological study (VEP, BAEP) in HIV-1 seropositive patients with and without AIDS. Acta Neurol Belg 1993;93:78-87.

35 Iragui VJ, Kalmijn J, Plummer DJ, et al. Pattern electroretinograms and visual evoked potentials in HIV infection: evidence of asymptomatic retinal and postretinal impairment in the absence of infectious retinopathy. Neurology 1996;47:1452-6.

36 Farnarier G, Somma-Mauvais H. Multimodal evoked potentials in HIV infected patients. Electroencephalogr Clin Neurophysiol 1990;41:355-69.

37 Smith T, Jakobsen J, Gaub J, et al. Clinical and electrophysiological studies of human immunodeficiency virus-seropositive men without AIDS. Ann Neurol 1988;23:295-7.

38 Sample PA, Plummer DJ, Mueller AJ, et al. Pattern of early visual field loss in HIV-infected patients. Arch Ophthalmol 1999;117:755-60.

39 Plummer DJ, Bartsch DU, Azen SP, et al. Retinal nerve fiber layer evaluation in human immunodeficiency virus-positive patients. Am J Ophthalmol 2001;131:216-22.

40 Tran Dinh YR, Mamo H, Cervoni J, et al. Disturbances in the cerebral perfusion of human immune deficiency virus-1 seropositive asymptomatic subjects: a quantitative tomography study of 18 cases. J Nucl Med 1990;31:1601-7. 